\title{
Disrupted Regulation of Social Exclusion in Alcohol-Dependence: An fMRI Study
}

\author{
Pierre Maurage*, 1,2,3, Frédéric Joassin ${ }^{1,2}$, Pierre Philippot ${ }^{2}$, Alexandre Heeren ${ }^{2,3}$, Nicolas Vermeulen ${ }^{2,3}$, \\ Pierre Mahau ${ }^{4}$, Christel Delperdange', Olivier Corneille ${ }^{3,5}$, Olivier Luminet ${ }^{2,3}$ and Philippe de Timary ${ }^{2,6,7}$ \\ 'Neuroscience, Systems and Cognition (NEUROCS) Research Unit, Institute of Psychology, Catholic University of Louvain, Louvain-la-Neuve, \\ Belgium; ${ }^{2}$ Health and Psychological Development (CSDP) Research Unit, Institute of Psychology, Catholic University of Louvain, Louvain-la-Neuve, \\ Belgium; ${ }^{3}$ Belgian Fund for Scientific Research, FRS-FNRS, Brussels, Belgium; ${ }^{4}$ Research Computer Science Unit, Institute of Psychology, Catholic \\ University of Louvain, Louvain-la-Neuve, Belgium; ${ }^{5}$ Social Behaviour (CECoS) Research Unit, Institute of Psychology, Catholic University of Louvain, \\ Louvain-la-Neuve, Belgium; ' Department of Adult Psychiatry, Saint-Luc University Hospital, Brussels, Belgium; ${ }^{7}$ Institute of Neuroscience, Catholic \\ University of Louvain, Brussels, Belgium
}

\begin{abstract}
Alcohol-dependence is associated with cognitive and biological alterations, and also with interpersonal impairments. Although overwhelming in clinical settings and involved in relapse, these social impairments have received little attention from researchers. Particularly, brain alterations related to social exclusion have not been explored in alcohol-dependence. Our primary purpose was to determine the neural correlates of social exclusion feelings in this population. In all, 44 participants (22 abstinent alcohol-dependent patients and 22 paired controls) played a virtual game ('cyberball') during fMRI recording. They were first included by other players, then excluded, and finally re-included. Brain areas involved in social exclusion were identified and the functional connectivity between these areas was explored using psycho-physiological interactions (PPI). Results showed that while both groups presented dorsal anterior cingulate cortex (dACC) activations during social exclusion, alcohol-dependent participants exhibited increased insula and reduced frontal activations (in ventrolateral prefrontal cortex) as compared with controls. Alcohol-dependence was also associated with persistent $\mathrm{dACC}$ and parahippocampal gyrus activations in re-inclusion. PPI analyses showed reduced frontocingulate connectivity during social exclusion in alcohol-dependence. Alcohol-dependence is thus linked with increased activation in areas eliciting social exclusion feelings (dACC-insula), and with impaired ability to inhibit these feelings (indexed by reduced frontal activations). Altered frontal regulation thus appears implied in the interpersonal alterations observed in alcohol-dependence, which seem reinforced by impaired frontocingulate connectivity. This first exploration of the neural correlates of interpersonal problems in alcohol-dependence could initiate the development of a social neuroscience of addictive states.

Neuropsychopharmacology (2012) 37, 2067-2075; doi:I0.1038/npp.2012.54; published online I8 April 2012
\end{abstract}

Keywords: social exclusion; ostracism; alcohol-dependence; fMRI

\section{INTRODUCTION}

Alcohol-dependence is the most widespread psychiatric disorder, constituting the third main preventable cause of mortality and costing US $\$ 180$ billion annually in the United States (US Department of Health and Human Services, 2000). Its cognitive and biological correlates have been explored extensively, leading to many therapeutic proposals (Johnson et al, 2003; Ross and Peselow, 2009), but the efficiency of alcohol-dependence treatment is still low, with

\footnotetext{
*Correspondence: Dr P Maurage, Institute of Psychological Sciences, Catholic University of Louvain, Place du Cardinal Mercier, I0, 1348 Louvain-la-Neuve, Belgium, Tel: + 3210 479245, Fax: + 3210473774 , E-mail: pierre.maurage@uclouvain.be

Received 2 February 2012; revised 14 March 2012; accepted 20 March 2012
}

a relapse rate around $60 \%$ within 6 months (Maisto et al, 2006). Recent proposals for improving detoxification underlined the need to use multimodal treatments combining pharmacotherapy with psychosocial interventions, and particularly to rehabilitate interpersonal impairments (Brandon et al, 2007; Swift, 2007), which constitute the main relapse factor after mid-term abstinence (Zywiak et al, 2003).

Being socially integrated is indeed a primary human need, but most mental illnesses lead to social alterations (Adolphs, 2010). This is particularly true for alcohol-dependence, which involves stronger interpersonal consequences (like public stigma and social exclusion) than other psychiatric states from both the general population and from primarycare providers (Nutt et al, 2010; Pescosolido et al, 2010). Ostracism (ie, the fact of being ignored or excluded by others) is thus regularly observed in alcohol-dependence 
and influences its development and maintenance (Schomerus et al, 2011). Although several interpersonal abilities (Uekermann and Daum, 2008) have been explored among alcohol-dependent subjects (ADS), ostracism has never been evaluated, and the neural correlates of interpersonal impairments remain totally unexplored.

The outcomes of social exclusion should thus be further investigated, and social neuroscience provides decisive opportunities in this perspective as innovative paradigms were recently developed to understand the biological mechanisms underlying social processes (Lieberman, 2007). The omnipresence of social impairments among neurological and psychiatric populations recently led social neuroscience to expand towards pathological states (Adolphs, 2010). The first clinical studies gave promising results, describing abnormal cortical and limbic activations during interpersonal processing in schizophrenia, depression, and autism (Sugranyes et al, 2011). Nevertheless, social exclusion has up to now only been explored in autism, where two studies showed reduced insula and dorsal anterior cingulate cortex (dACC) activations during ostracism (Bolling et al, 2011; Masten et al, 2011).

The primary aim of this study was to explore the neural correlates of social exclusion in alcohol-dependence. The cyberball task, a computerized ball-tossing game eliciting social exclusion feelings (Eisenberger et al, 2003), was used. fMRI studies (Eisenberger et al, 2003; Onoda et al, 2010; Gunther-Moor et al, 2012) have shown that the exclusion feelings evoked by this task are associated with two main cerebral activations: (a) dACC and insula activations, reflecting the negative feelings and distress associated with social exclusion; (b) middle frontal gyrus (MFG) and inferior frontal gyrus (IFG) activations, particularly in the right ventrolateral prefrontal cortex (VPFC, Brodmann's area 47), implicated in the regulation and inhibition of this emotional response. The high frequency of negative social interactions experienced by ADS leads to the hypothesis that they would present increased activations related to the elicitation of social exclusion feelings (ie, in dACC and insula) combined with reduced activations related to the regulation of these feelings (ie, in MFG and IFG). Finally, as earlier behavioral studies suggested that exclusion feelings persist in psychiatric populations even after exclusion ends (Zadro et al, 2006; Perry et al, 2011), we hypothesized that dACC activations would continue in alcohol-dependence after the exclusion period.

\section{MATERIALS AND METHODS}

\section{Participants}

In all, 22 male in-patients, diagnosed with alcoholdependence according to the DSM-IV criteria, were recruited during the third week of their detoxification treatment (Saint-Luc University Hospital, Brussels, Belgium). They had all abstained from alcohol for at least 14 days, were all right-handed, and were free of medication and of any other psychiatric diagnosis, as assessed by an exhaustive semi-structured psychiatric interview. The mean alcohol consumption among ADS just before detoxification was 19.6 drinks per day $(S D=10.59)$. The mean number of previous detoxification treatments was $3.8(\mathrm{SD}=5.3)$, and the mean duration of alcohol-dependence was 12.9 years $(\mathrm{SD}=10.2)$. Patients were matched for age, gender, and education with a control group composed of 22 male volunteers who were free of any personal or family history of psychiatric disorder, and whose personal alcohol consumption was lower than 15 drinks per week (mean daily consumption $=1.54(\mathrm{SD}=1.15))$, as assessed by an exhaustive semi-structured psychiatric interview. Control subjects (CS) abstained from any alcohol consumption during at least 3 days before testing. Exclusion criteria for both groups included major medical/neurological impairments, past head trauma, and polysubstance past or present abuse (including marijuana). Each participant had normal or corrected-to-normal vision. Education level was the number of years of education completed since starting primary school. After complete description of the study to participants, written informed consent was obtained. Participants were paid 40 euros for their participation. The study was approved by the ethical committee of the Catholic University of Louvain.

\section{Task and Procedure}

Blood oxygenation level-dependent signal changes were recorded during the Cyberball task (Williams and Jarvis, 2006; Williams, 2007), a virtual ball-tossing game in which participants were told they will be connected over the Intranet to play with other real players performing the task in other scanners. Actually, there were no other real players, participants played with computer-guided players. Participants were told that this experiment was about mental imagery and that the task was used to help them visualizing other players. This cover story ensured that participants believed the other players were real (Eisenberger et al, 2003). In the scanner, participants saw an animated ball-tossing game, with an icon representing their own hand at the bottom and the two other players depicted as animated icons in the upper corners. Players' names were written besides each player's icon. The Intranet connection status ('you are not connected' for the first condition, 'you are connected' for the three others) was written in the top left part. When receiving the ball, participants had $2.5 \mathrm{~s}$ to choose (using a two-button response pad) which player they wanted to give the ball to. Each participant took part in four successive conditions (Figure 1): (a) implicit social exclusion (ISE), where participants were told that the intranet connection was not effective yet because of technical problems, but that they could watch other participants playing; (b) first inclusion (INCL1), where participants were told they were connected and played with other players; (c) explicit social exclusion (ESE), where participants received five throws and were then excluded from the game (ie, other players started playing exclusively together, participant never received the ball anymore); and (d) second inclusion (INCL2), where participants were connected and re-included in the game. This fourth condition was added here to explore the brain correlates of re-inclusion after exclusion.

Except for the ISE-INCL1 switch (ie, from 'not connected' to 'connected'), participants were not informed of the transition between the successive conditions. Each condition lasted for $125 \mathrm{~s}$ (50 volumes). Computer players' speed varied randomly between $500 \mathrm{~ms}$ and $2 \mathrm{~s}$, and was adapted 

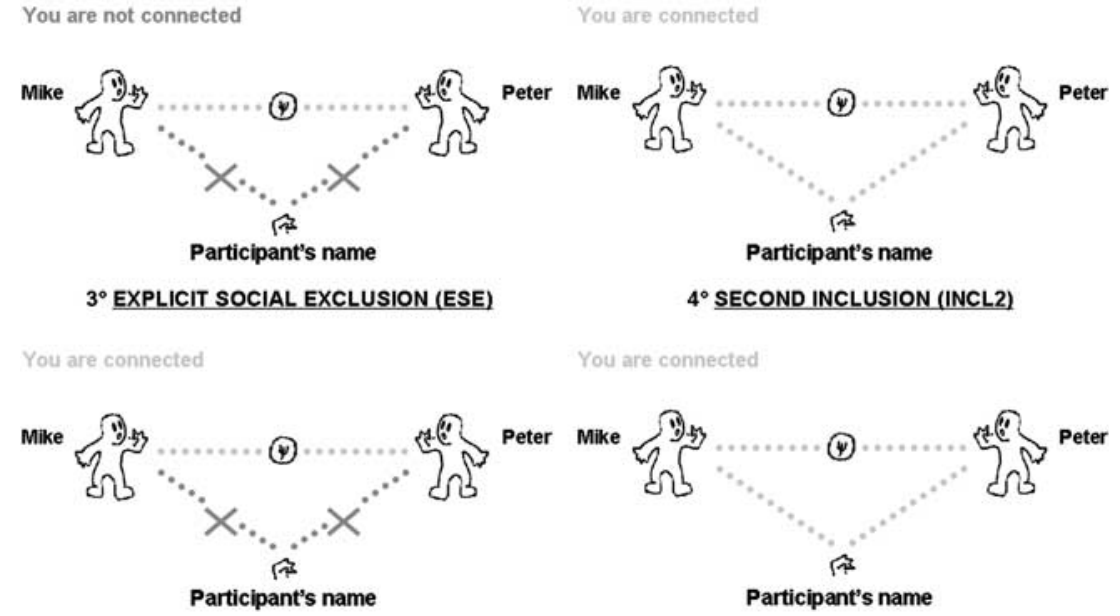

Figure I Illustration of the four successive experimental conditions: (I) implicit social exclusion (ISE); (2) first inclusion (INCLI); (3) explicit social exclusion (ESE), that is, the central experimental condition eliciting social exclusion feelings; and (4) second inclusion (INCL2), that is, re-inclusion after social exclusion.

to obtain 100 throws per condition. If the participant did not throw the ball within $2.5 \mathrm{~s}$, it was automatically thrown to a random player. The first 10 volumes of each condition were excluded from analyses (which relied on 40 volumes per condition) to avoid overlap between the activations associated with each condition. The two main experimental contrasts were: (a) ESE-ISE, isolating the cerebral correlates of social exclusion feelings, as ESE and ISE are perceptually identical (ie, other players excluding the participant, who never receives the ball), but differ for ostracism: in ISE, participant knows he is not participating because of technical reasons, whereas in ESE, he is explicitly excluded by other players, eliciting exclusion feelings; (b) INCL2INCL1, exploring the persistence of exclusion feelings after exclusion ends. INCL1 and INCL2 are perceptually identical (ie the participant being included, with a $50 \%$ probability of getting the ball), but in INCL1 the participant has not yet been explicitly excluded by others, whereas INCL2 is just following the ESE condition. Finally, to check the presence of exclusion feelings, participants answered (after the last condition) a manipulation check scale (from 1 'Absolutely not' to 7 'Extremely') assessing ostracism (ie 'I felt excluded by other participants').

\section{Imaging Procedure}

Functional images were acquired with $3 \mathrm{~T}$ magnetic resonance imager and 8-channel phased array head coil (Achieva, Philips Medical Systems) as series of blood-oxygen-sensitive $\mathrm{T} 2^{*}$ weighted echo-planar image volumes. Acquisition parameters were $\mathrm{TE}=32 \mathrm{~ms}, \mathrm{TR}=2500 \mathrm{~ms}$, flip angle $=90^{\circ}, \mathrm{FOV}=220$ $\times 220 \mathrm{~mm}^{2}$, slice thickness $=3.5 \mathrm{~mm}$ with no interslice gap, and SENSE factor $=2.5$. Each volume comprised 36 axial slices acquired in ascending interleaved sequence. Recording comprised one 208 volumes run ( 50 volumes per condition, interleaved by 2 volumes transition periods). High-resolution anatomical images were also acquired using a T1-weighted 3D turbo fast field echo sequence with inversion recovery prepulse (150 contiguous $1 \mathrm{~mm}$ axial slices, $\mathrm{TE}=4.6 \mathrm{~ms}$,
$\mathrm{TR}=9.1 \mathrm{~ms}$, flip angle $=8^{\circ}, \mathrm{FOV}=220 \times 197 \mathrm{~mm}^{2}$, voxel size $=0.81 \times 0.95 \times 1 \mathrm{~mm}^{3}$, and SENSE factor $=1.4$ ).

\section{fMRI Data Analysis and Statistical Analysis}

Data were processed using Statistical Parametric Mapping (SPM8, Welcome Department of Cognitive Neurology, UK), implemented in Matlab 7.1 (Mathworks). Functional images were corrected for slice acquisition delays; realigned to the first scan to correct within- and between-run motion; coregistered with anatomical scan; normalized to the MNI template using an affine fourth-degree $\beta$-spline interpolation transformation and a voxel size of $2 \times 2 \times 2 \mathrm{~mm}^{3}$ after the skull and bones had been removed with a mask based on individual anatomical images; and spatially smoothed using a $10 \mathrm{~mm}$ FWHM Gaussian kernel.

Condition-related changes in regional brain activity were estimated for each participant by a general linear model in which the responses evoked by each condition were modeled by a standard hemodynamic response function. Two main contrasts of interest were computed at the individual level: (a) explicit exclusion as compared with implicit exclusion (ESE-ISE); (b) re-inclusion as compared with initial inclusion (INCL2-INCL1). Significant cerebral activations were then examined at the group level in random-effect analyses using one-sample $t$-tests, with statistical threshold set to $p<0.05$ FWE corrected for multiple comparisons using cluster size and extending to at least 20 contiguous voxels. Between-group comparisons were conducted using two-sample $t$-tests with the same statistical threshold.

A psychophysiological interaction (PPI) analysis (Friston et al, 1997; Friston, 2004) determined regions functionally connected with dACC during ESE-ISE contrast. For each participant, a region of interest (ROI) was determined $(5 \mathrm{~mm}$ radius sphere centered on group maximum activity peak in dACC), and the deconvolved activity time course in this ROI was extracted. Activity time course was corrected for the effect of interest. The product of this activation time course was calculated with a condition-specific regressor probing the social exclusion (ESE-ISE) to create PPI terms. 
PPI analyses was conducted for each participant and entered into a random-effects analysis for each group with onesample $t$-tests, using a statistical threshold of $p<0.001$ uncorrected with a cluster threshold of 20 contiguous voxels. The estimated connectivity was represented as a $t$-value, and the positive $t$-value representing positive interaction with dACC (and conversely for negative values). Between-group comparisons were conducted using two-sample $t$-tests with the same statistical threshold. Finally, for each area significantly activated in each group during ESE-ISE contrast, a ROI was determined ( $5 \mathrm{~mm}$ radius sphere centered on group maximum activity peak in this area), and mean $\beta$ value for ROI was extracted to perform Pearson's correlations between these areas and behavioral results. These complementary analyses were conducted following recent recommendations improving the validity of the correlations between behavioral and neuroimaging data (Poldrack and Mumford, 2009; Vul et al, 2009), to avoid the overestimation of correlations due to the non-independence error.

\section{RESULTS}

\section{Control Measures and Exclusion Feelings Scale}

One-way analyses of variance showed that groups did not significantly differ for age (mean $\mathrm{ADS}=47.2(\mathrm{SD}=11.04)$; mean $C S=45.1(S D=10.69) ; \mathrm{F}(1,42)=0.41, \mathrm{NS})$ and education (mean $\mathrm{ADS}=13.91 \quad(\mathrm{SD}=3.53)$; mean $\mathrm{CS}=14.59$ $(\mathrm{SD}=3.24) ; \mathrm{F}(1,42)=0.44, \mathrm{NS})$.

Post-experiment manipulation check confirmed that the task elicited social exclusion, as participants reported marked exclusion feelings (significantly higher than minimal score indexing no exclusion, $\mathrm{t}(43)=12.45, p<0.001)$. The mean exclusion feelings score was $3.73(\mathrm{SD}=1.61)$ among $\mathrm{ADS}$ and $3.59(\mathrm{SD}=1.73)$ among $\mathrm{CS}$, with no significant group difference $(\mathrm{F}(2,42)=0.069, \mathrm{NS})$.

\section{fMRI Data}

Social exclusion. The ESE-ISE contrast showed the brain activations associated with social exclusion feelings (Table $1 \mathrm{a}$ and $\mathrm{c}$ and Figure 2):

- CS: Significant activations were found in the right VPFC, superior frontal gyrus (SFG), and insula, and in the left $\mathrm{dACC}$, posterior cingulate cortex (PCC), MFG, and superior temporal gyrus (STG).

- ADS: Significant activations were found in the right dACC, insula, precentral gyrus, and IFG, and in the left PCC.

- Group comparison: Social exclusion elicited more right insula activation among ADS than CS, whereas CS had higher right VPFC and left MFG activations than ADS.

Re-inclusion after social exclusion. The INCL2-INCL1 contrast showed the brain areas activated during reinclusion after social exclusion, as compared with first inclusion period (Table $1 \mathrm{~b}$ and $\mathrm{c}$ and Figure 2):

- CS: No significant activations were found.

- ADS: Significant activations were found in the left dACC and parahippocampal gyrus (PHG).
- Group comparison: Re-inclusion after exclusion led to higher left dACC and PHG activations among ADS than among CS. Finally, no significant group differences were found for contrasts comparing inclusion conditions with the non-inclusion baseline (ie INCL1-ISE, INCL2-ISE) nor for the INCL1-INCL2 contrast.

\section{Correlational analyses}

- Behavioral-fMRI correlations: The intensity of subjective exclusion feelings (evaluated by the post-experiment scale) was positively correlated with dACC activation in both groups (CS: $r=0.528, p<0.05$; ADS: $r=0.633$, $<0.01)$. Moreover, subjective exclusion feelings were (a) positively correlated with insula activation among ADS $(r=0.431, p<0.05)$, but not CS $(r=0.168$, NS); (b) negatively correlated with VPFC among CS $(r=-0.495$, $p<0.05)$, but not ADS $(r=0.224$, NS).

- Correlations between brain areas: In the control group, significant positive correlations were found between dACC, insula, and PCC (dACC-insula: $r=0.788, p<0.001$; dACCPCC: $r=0.461, p<0.05$; PCC-insula: $r=0.756, p<0.001$ ). Moreover, significant negative correlations were found between dACC, insula, and MFG (dACC-MFG: $r=-0.593$, $p<0.01$; insula-MFG: $r=-0.659, p<0.001)$, and between VPFC and PCC $(r=-0.505, p<0.01)$. In the alcoholdependent group, significant positive correlations were found between dACC, insula, and PCC (dACC-insula: $r=0.557, p<0.01$; dACC-PCC: $r=0.759, p<0.001$; PCCinsula: $r=0.455, p<0.05)$, but no significant correlations appeared with frontal areas.

PPI during social exclusion. PPI showed functional connectivity with dACC during social exclusion (Table 2):

- CS: dACC presented a significant positive connectivity with bilateral STG, and a significant negative connectivity with right VPFC, IFG, and SFG, and with left MFG.

- ADS: dACC presented a significant positive connectivity with left PHG and right STG, and a significant negative connectivity with left postcentral gyrus.

- Group comparison: Positive connectivity was higher among CS in right STG and in left thalamus, precentral gyrus, and postcentral gyrus. Negative connectivity was higher among CS in bilateral MFG, IFG, SFG, and VPFC.

\section{DISCUSSION}

Our study identified for the first time the cerebral correlates of interpersonal alterations in alcohol-dependence. Social exclusion constitutes a major relapse factor in alcoholdependence (Zywiak et al, 2003; Pescosolido et al, 2010), but it had not yet been experimentally studied, and the cerebral alterations associated with interpersonal deficits remained totally unexplored. First, the manipulation check confirmed that both groups experienced social exclusion, with similar intensities than in previous studies (Eisenberger et al, 2003; Masten et al, 2011). The absence of group differences is in line with earlier clinical results (Bolling et al, 2011; Masten et al, 2011; Perry et al, 2011) and underlines that, while brain activations are strongly modified in psychiatric populations 
Table I Significant Brain Activations for (a) Social Exclusion as Compared with Implicit Exclusion (ESE-ISE) Among CS and ADS; (b) ReInclusion After Social Exclusion as Compared with First Inclusion (INCL2-INCLI) in Each Group; and (c) Group Comparison During Social Exclusion and Re-Inclusion

(a) ESE-ISE

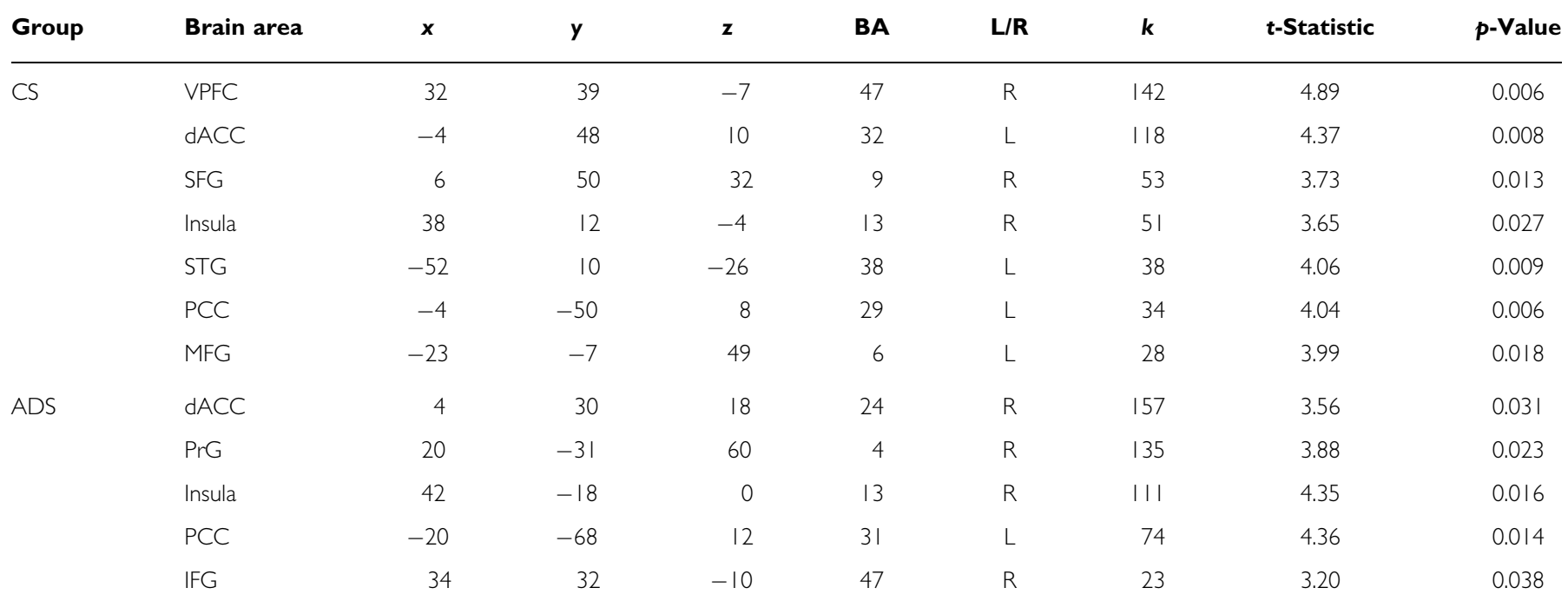

(b) INCL2-INCLI

\begin{tabular}{|c|c|c|c|c|c|c|c|c|c|}
\hline Group & Brain area & $x$ & $y$ & $z$ & BA & L/R & $k$ & t-Statistic & $p$-Value \\
\hline \multirow[t]{2}{*}{ ADS } & PHG & -24 & -52 & 0 & 19 & L & 245 & 4.01 & 0.027 \\
\hline & $\mathrm{dACC}$ & -12 & 40 & 14 & 32 & L & 57 & 3.41 & 0.039 \\
\hline
\end{tabular}

(c) Group comparison

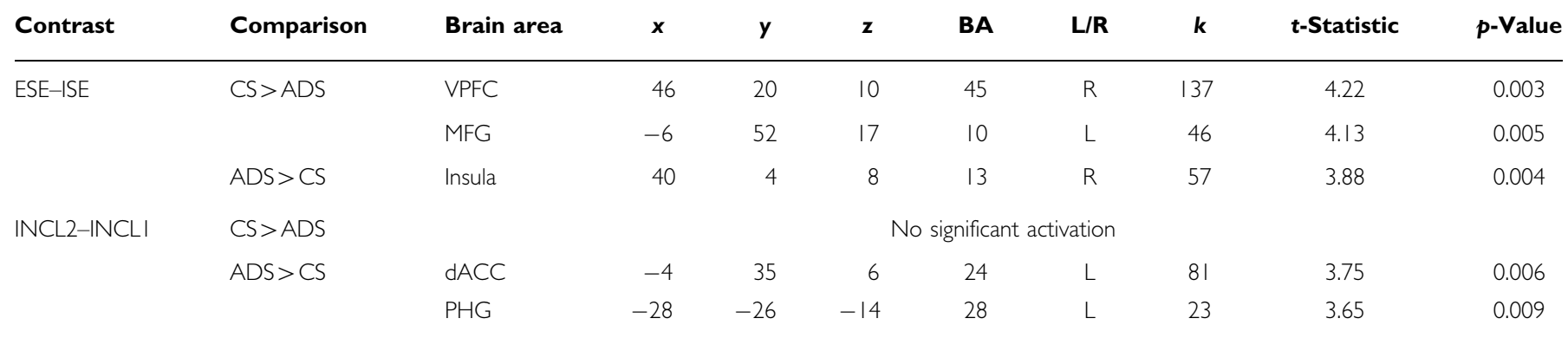

Abbreviations: ADS, alcohol-dependent subjects; BA, Brodmann area; CS, control subjects; dACC, dorsal anterior cingulate cortex; IFG, inferior frontal gyrus; $k$, cluster size; L, left hemisphere; MFG, middle frontal Gyrus; PCC, posterior cingulate cortex; PHG, parahippocampal gyrus; PrG, precentral gyrus; R, right hemisphere; SFG, superior frontal gyrus; STG, superior temporal gyrus; VPFC, ventrolateral prefrontal cortex. Threshold set at $p<0.05, p$-values are FWE corrected for multiple comparisons using cluster size, with a minimum cluster size of 20 voxels.

$x, y$, and $z$ are stereotaxic coordinates of peak-height voxels.

during social exclusion, these populations describe comparable subjective exclusion feelings than controls. This observation reinforces the proposal (Eisenberger and Lieberman, 2004) that neuroimaging techniques are needed to explore precisely interpersonal processes unreachable by subjective evaluation, particularly in populations like ADS, characterized by altered ability to identify correctly their own affective states and by higher social desirability bias (Uekermann and Daum, 2008).
Concerning brain activations, the CS results confirm earlier studies (Eisenberger et al, 2003; Eisenberger and Lieberman, 2004), as the classical activation pattern related to social exclusion was observed: (a) dACC and insula activations are usually interpreted as being related to social exclusion perception and associated affective distress (Krill and Platek, 2009; Onoda et al, 2010; Bolling et al, 2011; Masten et al, 2011). Although the correlational analyses between behavioral and neuroimaging data have been 


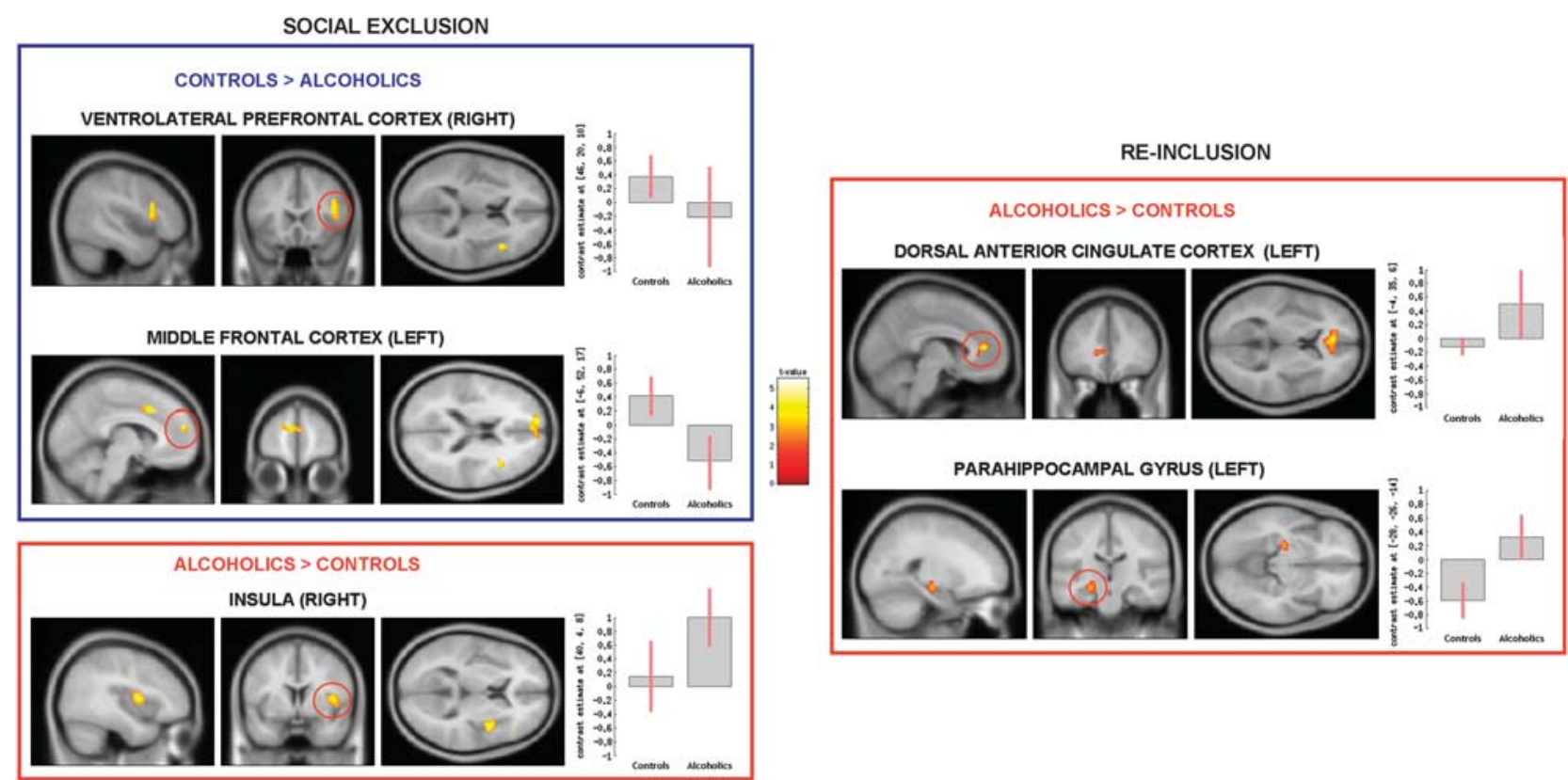

Figure 2 Group comparison for social exclusion (explicit social exclusion (ESE)-implicit social exclusion (ISE), on the left) and re-inclusion (second inclusion (INCL2)-first inclusion (INCLI), on the right) contrasts, showing the brain areas presenting significantly reduced (in blue) or increased (in red) activations among alcohol-dependent participants $(N=22)$ as compared with controls $(N=22) ; p<0.05$ corrected for multiple comparisons at cluster size. $\beta$-Values for each group in each brain area are presented in graphs on the right; error bars indicate standard deviations.

recently criticized (Vul et al, 2009), these results are reinforced by the correlations observed between dACC activation and subjective exclusion feelings, as these correlational analyses have been conducted following the recommendations made to avoid the non-independence error (Poldrack and Mumford, 2009; Vul et al, 2009); (b) frontal area activations (right VPFC-MFG-SFG) are associated with the regulation of social pain and affective distress inhibition (Eisenberger and Lieberman, 2004). This frontal regulation is confirmed by the negative frontal-dACC correlations and by PPI analyses showing negative functional connectivity between dACC and frontal areas (VPFCIFG-MFG-SFG). Our results thus reinforce previous ones with sounder methodology, as FWE-corrected thresholds were applied here while most previous results (Eisenberger et al, 2003; DeWall et al, 2010; Onoda et al, 2010; Bolling et al, 2011; Masten et al, 2011) relied on uncorrected thresholds. Moreover, the re-inclusion condition (INCL2) extends earlier results by suggesting that $\mathrm{AACC}$ and frontal activations are directly linked with exclusion feelings, as these activations rapidly disappear among CS when exclusion ends (no activation was found in this group for INCL2INCL1).

Importantly, ADS showed modified brain activations during ostracism as compared with CS. First, ESE-ISE contrast revealed: (1) increased insula activity, classically interpreted as indexing a higher emotional reaction to ostracism (Eisenberger et al, 2003; Onoda et al, 2010; Bolling et al, 2011; Masten et al, 2011; Gunther-Moor et al, 2012). Nevertheless, while the link between insula and exclusion feelings has been repeatedly proposed in earlier studies, this increased insula activation during social exclusion among ADS could also partly be due to increased negative emotions rather than to enhanced social exclusion feelings per se. Indeed, earlier studies have shown that ADS present increased insula activation during non-social negative emotional situations, as compared with CS (Bjork et al, 2008; Gilman et al, 2010). The specificity of this insula activation for the social nature of the task can thus not be totally proven on the basis of the present results; (2) reduced right VPFC-MFG activations, suggesting an impaired inhibition of social exclusion feelings (Eisenberger et al, 2003; Onoda et al, 2010; Bolling et al, 2011; Masten et al, 2011; Gunther-Moor et al, 2012). This is reinforced by the fact that the negative correlations observed between dACC, insula, and PCC, and frontal regions among CS were absent among ADS. Reduced VPFC activation during ostracism had been described in autism (Bolling et al, 2011; Masten et al, 2011), and this inability to regulate social exclusion could be common to different psychiatric states.

Second, ADS presented increased dACC and PHG activations in INCL2-INCL1 contrast, suggesting the persistence of exclusion feelings even after re-inclusion. This persistent social distress, observed behaviorally in schizophrenia, may be linked to the appearance of negative ruminations after exclusion (Zadro et al, 2006; Perry et al, 2011). PHG overactivation reinforces the hypothesis of an increased sensitivity to social exclusion in alcohol-dependence, as several studies described PHG activations during ostracism (Bolling et al, 2010; DeWall et al, 2010; Onoda et al, 2010) and as overactivation of this area, involved in visual memory, reflects deeper encoding of exclusion stimulations (Kross et al, 2007). It should also be noted that the dACC activations partly extend towards the pregenual ACC. As this region is known to be highly activated during physical pain regulation (Zubieta et al, 2005; Tracey, 2010) and during stressful situations with emotional content (Phan et al, 2002), these pregenual ACC 
Table 2 Brain Areas Presenting Significant Functional Connectivity with dACC During Social Exclusion (ESE-ISE contrast), as Shown by PPI Analysis for (a) CS and ADS; (b) Group Comparison

(a)

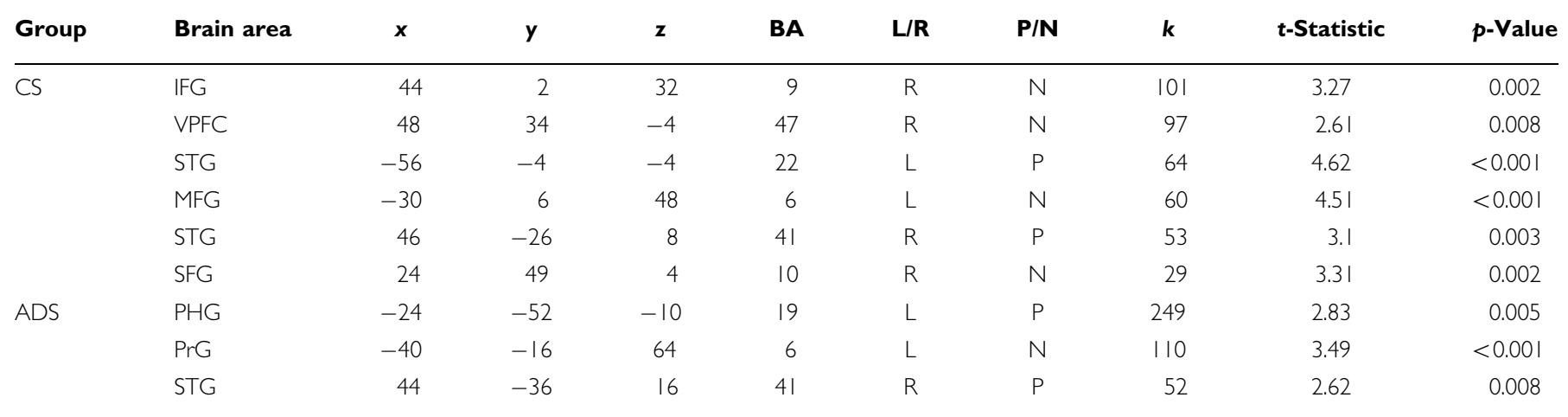

(b)

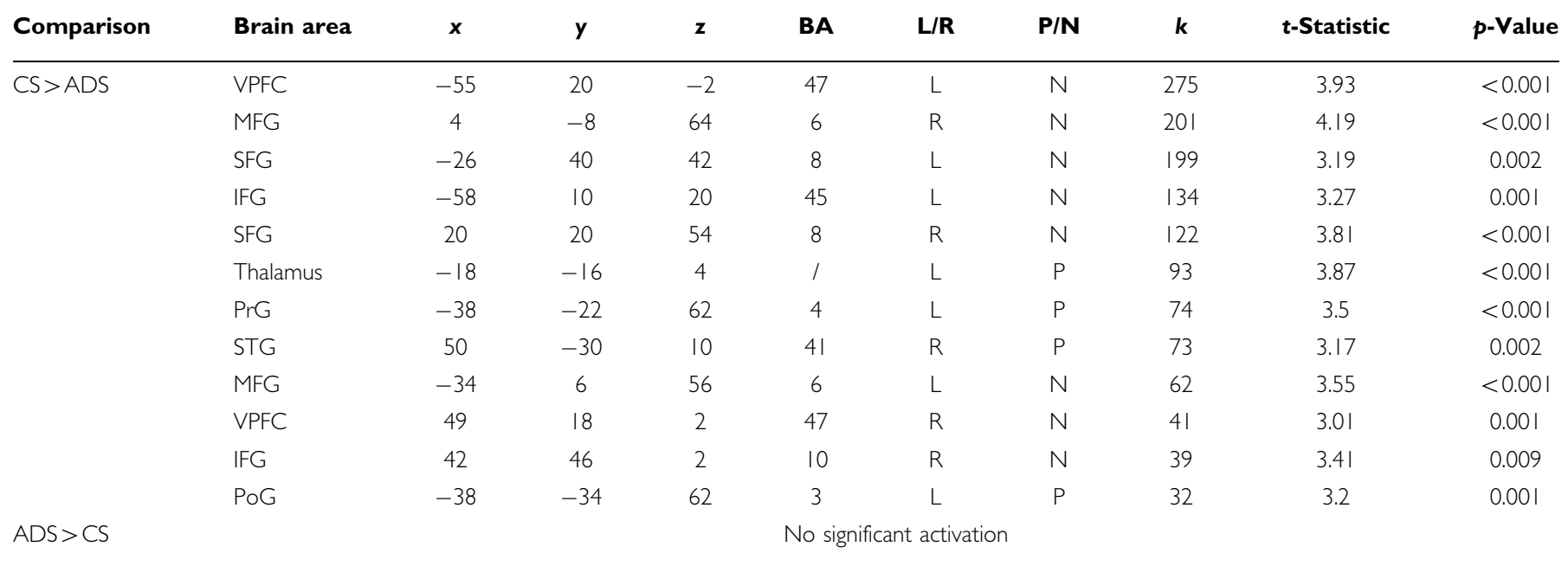

Abbreviation: ADS, alcohol-dependent subjects; BA, Brodmann area; CS, control subjects; dACC, dorsal anterior cingulate cortex; IFG, inferior frontal fyrus; $k$, cluster size; L, left hemisphere; MFG, middle frontal gyrus; N, negative connectivity; P, positive connectivity; PHG, parahippocampal gyrus; PoG, postcentral gyrus; PrG, precentral gyrus; R, right hemisphere; SFG, superior frontal gyrus; STG, superior temporal gyrus; VPFC, ventrolateral prefrontal cortex.

Threshold set at $p<0.00$ I uncorrected with a minimum cluster size of 20 voxels.

$x, y$, and $z$ are stereotaxic coordinates of peak-height voxels.

activations during re-inclusion reinforce the hypothesis of a persistent social pain among ADS. However, as underlined above, this study did not include a control non-social condition and these enhanced AACC and PHG activations among ADS could thus also partly be due to negative emotions related with exclusion rather than to persistent social exclusion feelings themselves. Finally, PPI analyses confirmed the reduced frontal regulation among ADS, as the negative frontocingulate functional connectivity observed among CS is absent among ADS. Taken together, these results strongly suggest that alcohol-dependence is associated with increased activation of the cerebral network related to social exclusion feelings (ie dACC, insula, and PHG overactivations during and after social exclusion) and reduced the ability to regulate them (ie reduced frontal activations and impaired dACC-frontal functional connectivity). These results appear specifically related to social exclusion, as no group differences were found in other contrasts. It should, however, be noted that, in line with earlier studies based on this paradigm, the present one did not include a control non-social experimental condition, and future studies will thus have to confirm that the cerebral alterations observed here among ADS are indeed related to the social processes involved in the task and not to more general factors like increased negative emotions.

Although these results will have to be extended to a more general population (particularly ADS presenting comorbidities like polysubstance abuse or depression-anxiety disorders, which are frequent in alcohol-dependence (eg, Sintov et al, 2010)), they already have several direct implications. At the theoretical level, they extend the 'frontal lobe hypothesis' to interpersonal problems. This hypothesis states that the cognitive impairments in alcoholdependence are mainly due to frontal alterations (Moselhy et al, 2001). It had been suggested that frontal alterations might also be responsible for social deficits of ADS (Uekermann and Daum, 2008), but no experimental results had confirmed this proposal. Our data clearly support it by 
suggesting that interpersonal disturbances are linked with an inability to correctly use frontal inhibitory control for social distress regulation. Moreover, we showed that this inability relies on two cumulative impairments: (a) reduced frontal activations (VPFC-MFG) during ostracism, which is in line with previous studies determining anatomical and functional frontal alterations in alcohol-dependence (Bühler and Mann, 2011). It has recently been shown that the extent of these frontal alterations is correlated with higher relapse probability (Rando et al, 2011). As social disturbances are highly involved in relapse (Zywiak et al, 2003; Uekermann and Daum, 2008), our results suggest that this link between frontal alterations and relapse could be partly due to this inability to regulate social problems when frontal areas are damaged; (b) altered frontocingulate functional connectivity. Several studies showed white matter abnormalities in alcohol-dependence (Pfefferbaum and Sullivan, 2005), which are correlated with higher relapse rates (Sorg et al, 2012). This study, being among the first ones to use PPI analyses in alcohol-dependence, extends these previous results by identifying the functional correlates of structural white matter impairments: these impairments notably lead to altered frontocingulate connectivity, hampering the cognitive regulation of social exclusion feelings and potentially increasing relapse risk.

At the clinical level, our results, combined with the strong social stigma elicited by alcohol-dependence (Nutt et al, 2010; Pescosolido et al, 2010), first suggest that the treatment access difficulties in alcohol-dependence (Bijl and Ravelli, 2000) might be partly due to increased exclusion feelings. This should encourage physicians to adapt their interactions with patients and to promote outreach directed perspectives (Saunders et al, 2006). Secondly, increased dACC activations during interpersonal situations extend earlier results showing that dACC overactivations play a prominent role in emotional difficulties and increase relapse risk (Heinz et al, 2007; Vollstädt-Klein et al, 2010). As we suggested that these dACC overactivations are related to a lack of frontal inhibition, therapeutic programs increasing the ability to regulate interpersonal emotions constitute a promising therapeutic challenge. Such empirically validated social rehabilitation programs are used in schizophrenia (Veltro et al, 2011), but not yet in alcohol-dependence. More specifically, it has been shown that low self-esteem increases social distress during ostracism, this higher sensitivity being associated with reduced frontal regulation and frontocingulate connectivity (Onoda et al, 2010). The parallelism between these results and ours suggest that social difficulties could be partly due to reduced self-esteem in ADS (Trucco et al, 2007). Increasing self-esteem could thus reduce interpersonal deficits and relapse risk. To sum up, rehabilitation programs focusing on interpersonal abilities should be developed in alcohol-dependence. Finally, a crucial implication of this study is to show that social neuroscience can be used among ADS to develop innovative theoretical and therapeutic perspectives. Neural correlates of social cognition have recently been explored in schizophrenia and autism (Sugranyes et al, 2011), and we underlined the interest of applying social neuroscience paradigms to alcohol-dependence.

In sum, our main contribution is to show that social exclusion feelings in alcohol-dependence are associated with: (a) increased activations in areas usually associated with social exclusion feelings (dACC, insula, and PHG), during and after exclusion; (b) reduced activations of the frontal areas regulating these feelings. This suggests that interpersonal difficulties rely on a double brain alteration: overactivations in areas related to exclusion feelings and underactivations in areas regulating these feelings. This imbalance impairs social exclusion regulation, the impaired frontocingulate connectivity further increasing this impairment. These results offer the first insights concerning the cerebral correlates of interpersonal problems in alcoholdependence, and could constitute a first step towards the development of a social neuroscience of addictive states.

\section{ACKNOWLEDGEMENTS}

Pierre Maurage (Senior Research Fellow), Alexandre Heeren (Research Fellow), Nicolas Vermeulen (Research Associate), Olivier Corneille, Olivier Luminet (Senior Research Associate), and Philippe de Timary (Clinical Research Associate) are founded by the Belgian Fund for Scientific Research (FRS - FNRS, Belgium). This founding organization did not exert any role, editorial direction, or censorship in this experiment. We thank Professor Mauro Pesenti and Valérie Dormal for their help in data acquisition and analyses.

\section{DISCLOSURE}

The authors declare no conflict of interest.

\section{REFERENCES}

Adolphs R (2010). Conceptual challenges and directions for social neuroscience. Neuron 65: 752-767.

Bijl RV, Ravelli A (2000). Psychiatric morbidity, service use, and need for care in the general population: results of The Netherlands Mental Health Survey and Incidence Study. Am J Public Health 90: 602-607.

Bjork JM, Smith AR, Hommer DW (2008). Striatal sensitivity to reward deliveries and omissions in substance dependent patients. NeuroImage 42: 1609-1621.

Bolling DZ, Pitskel NB, Deen B, Crowley MJ, McPartland JC, Kaiser MD et al (2011). Enhanced neural responses to rule violation in children with autism: a comparison to social exclusion. Dev Cogn Neurosci 1: 280-294.

Bolling DZ, Pitskel NB, Deen B, Crowley MJ, McPartland JC, Mayes LC et al (2010). Dissociable brain mechanisms for processing social exclusion and rule violation. NeuroImage 54: 2462-2471.

Brandon TH, Vidrine JI, Litvin EB (2007). Relapse and relapse prevention. Annu Rev Clin Psychol 3: 257-284.

Bühler M, Mann K (2011). Alcohol and the human brain: a systematic review of different neuroimaging methods. Alcohol Clin Exp Res 35: 1771-1793.

DeWall CN, Macdonald G, Webster GD, Masten CL, Baumeister RF, Powell C et al (2010). Acetaminophen reduces social pain: behavioral and neural evidence. Psychol Sci 21: 931-937.

Eisenberger NI, Lieberman MD (2004). Why rejection hurts. A common neural alarm system for physical and social pain. Trends Cogn Sci 8: 294-300.

Eisenberger NI, Lieberman MD, Williams KD (2003). Does rejection hurt? An fMRI study of social exclusion. Science 302: 290-292.

Friston KJ (2004). Functional and effective connectivity in neuroimaging: a synthesis. Hum Brain Mapp 2: 56-78. 
Friston KJ, Buechel C, Fink GR, Morris J, Rolls E, Dolan RJ (1997). Psychophysiological and modulatory interactions in neuroimaging. NeuroImage 6: 218-229.

Gilman JM, Davis MB, Hommer DW (2010). Greater activation in left hemisphere language-related regions during simple judgment tasks among substance-dependent patients in treatment for alcoholism. Alcohol Clin Exp Res 34: 331-341.

Gunther-Moor B, Güroğlu B, Op de Macks ZA, Rombouts SA, Van der Molen MW, Crone EA (2012). Social exclusion and punishment of excluders: neural correlates and developmental trajectories. NeuroImage 59: 708-717.

Heinz A, Wrase J, Kahnt T, Beck A, Bromand Z, Grüsser SM et al (2007). Brain activation elicited by affectively positive stimuli is associated with a lower risk of relapse in detoxified alcoholic subjects. Alcohol Clin Exp Res 31: 1138-1147.

Johnson B, Ruiz P, Galanter M (2003). Handbook of Clinical Alcoholism Treatment, 1st edn. Lippincott Williams \& Wilkins: Philadelphia, PA.

Krill A, Platek SM (2009). In-group and out-group membership mediates anterior cingulate activation to social exclusion. Front Evol Neurosci 1: 1-7.

Kross E, Egner T, Ochsner K, Hirsch J, Downey G (2007). Neural dynamics of rejection sensitivity. J Cogn Neurosci 19: 945-956.

Lieberman MD (2007). Social cognitive neuroscience: a review of core processes. Annu Rev Psychol 58: 259-289.

Maisto SA, Zywiak WH, Connors GJ (2006). Course of functioning 1 year following admission for treatment of alcohol use disorders. Addict Behav 31: 69-79.

Masten CL, Colich NL, Rudie JD, Bookheimer SY, Eisenberger NI, Dapretto M (2011). An fMRI investigation of responses to peer rejection in adolescents with autism spectrum disorders. Dev Cogn Neurosci 1: 260-270.

Moselhy HF, Georgiou G, Kahn A (2001). Frontal lobe changes in alcoholism: a review of the literature. Alcohol Alcohol 36: 357-368.

Nutt DJ, King LA, Phillips LD, Independent Scientific Committee on Drugs (2010). Drug harms in the UK: a multicriteria decision analysis. Lancet 376: 1558-1565.

Onoda K, Okamoto Y, Nakashima K, Nittono H, Yoshimura S, Yamawaki $S$ et al (2010). Does low self-esteem enhance social pain? The relationship between trait self-esteem and anterior cingulate cortex activation induced by ostracism. Soc Cogn Affect Neurosci 5: 385-391.

Perry Y, Henry JD, Sethi N, Grisham JR (2011). The pain persists: how social exclusion affects individuals with schizophrenia. $\mathrm{Br} \mathrm{J}$ Clin Psychol 50: 339-349.

Pescosolido BA, Martin JK, Long JS, Medina TR, Phelan JC, Link BG (2010). 'A disease like any other'? A decade of change in public reactions to schizophrenia, depression, and alcohol dependence. Am J Psychiatry 167: 1321-1330.

Pfefferbaum A, Sullivan EV (2005). Disruption of brain white matter microstructure by excessive intracellular and extracellular fluid in alcoholism: evidence from diffusion tensor imaging. Neuropsychopharmacology 30: 423-432.

Phan KL, Wager T, Taylor SF, Liberzon I (2002). Functional neuroanatomy of emotion: a meta-analysis of emotion activation studies in PET and fMRI. NeuroImage 16: 331-348.

Poldrack RA, Mumford JA (2009). Independence in ROI analysis: where is the voodoo? Soc Cogn Affect Neurosci 4: 208-213.

Rando K, Hong KI, Bhagwagar Z, Ray Li CS, Bergquist K, Guarnaccia J et al (2011). Association of frontal and posterior cortical gray matter volume with time to alcohol relapse: a prospective study. Am J Psychiatry 168: 183-192.

Ross S, Peselow E (2009). Pharmacotherapy of addictive disorders. Clin Neuropharmacol 32: 277-289.

Saunders SM, Zygowicz KM, D'Angelo BR (2006). Person-related and treatment-related barriers to alcohol treatment. J Subst Abuse Treat 30: 261-270.

Schomerus G, Lucht M, Holzinger A, Matschinger H, Carta MG, Angermeyer MC (2011). The stigma of alcohol dependence compared with other mental disorders: a review of population studies. Alcohol Alcohol 46: 105-112.

Sintov ND, Kendler KS, Young-Wolff KC, Walsh D, Patterson DG, Prescott CA (2010). Empirically defined subtypes of alcohol dependence in an Irish family sample. Drug Alcohol Depend 107: 230-236.

Sorg SF, Taylor MJ, Alhassoon OM, Gongvatana A, Theilmann RJ, Frank LR et al (2012). Frontal white matter integrity predictors of adult alcohol treatment outcome. Biol Psychiatry 71: 262-268.

Sugranyes G, Kyriakopoulos M, Corrigall R, Taylor E, Frangou S (2011). Autism spectrum disorders and schizophrenia: metaanalysis of the neural correlates of social cognition. PLoS One 6: e25322.

Swift R (2007). Emerging approaches to managing alcohol dependence. Am J Health Syst Pharm 64: 12-22.

Tracey I (2010). Getting the pain you expect: mechanisms of placebo, nocebo and reappraisal effects in humans. Nat Med 16: 1277-1283.

Trucco EM, Connery HS, Griffin ML, Greenfield SF (2007). The relationship of self-esteem and self-efficacy to treatment outcomes of alcohol-dependent men and women. Am J Addict 16: 85-92.

US Department of Health and Human Services (2000). 10th Special Report to the US Congress on Alcohol and Health, 1st edn. National Institute on Alcohol Abuse and Alcoholism: Bethesda, MD.

Uekermann J, Daum I (2008). Social cognition in alcoholism: a link to prefrontal cortex dysfunction? Addiction 103: 726-735.

Veltro F, Mazza M, Vendittelli N, Alberti M, Casacchia M, Roncone $R$ (2011). A comparison of the effectiveness of problem solving training and of cognitive-emotional rehabilitation on neurocognition, social cognition and social functioning in people with schizophrenia. Clin Pract Epidemiol Ment Health 7: 123-132.

Vollstädt-Klein S, Hermann D, Rabinstein J, Wichert S, Klein O, Ende $\mathrm{G}$ et al (2010). Increased activation of the ACC during a spatial working memory task in alcohol-dependence versus heavy social drinking. Alcohol Clin Exp Res 34: 771-776.

Vul E, Harris C, Winkielman P, Pashler H (2009). Puzzlingly high correlations in fMRI studies of emotion, personality and social cognition. Perspect Psychol Sci 4: 274-290.

Williams KD (2007). Ostracism. Annu Rev Psychol 58: 425-452.

Williams KD, Jarvis B (2006). Cyberball: a program for use in research on interpersonal ostracism and acceptance. Behav Res Methods 38: 174-180.

Zadro L, Boland C, Richardson R (2006). How long does it last? The persistence of the effects of ostracism in the socially anxious. J Exp Soc Psychol 42: 692-697.

Zubieta JK, Bueller JA, Jackson LR, Scott DJ, Xu Y, Koeppe RA et al (2005). Placebo effects mediated by endogenous opioid activity on mu-opioid receptors. J Neurosci 25: 7754-7762.

Zywiak WH, Westerberg VS, Connors GJ, Maisto SA (2003). Exploratory findings from the reasons for drinking questionnaire. J Subst Abuse Treat 25: 287-292. 